\begin{tabular}{|c|c|}
\hline Title & V isualization of electric fields around a biological body \\
\hline Author(s) & Shimizu, K.; Endo, H.; Matsumoto, G. \\
\hline Citation & $\begin{array}{l}\text { IEEE Transactions on Biomedical Engineering, 35(5), 296-302 } \\
\text { https://doi.org/10.1109/10.1384 }\end{array}$ \\
\hline Issue Date & $1988-05$ \\
\hline Doc URL & http:/hdl.handle.net/2115/6046 \\
\hline Rights & $\begin{array}{l}\text { (C1988 IEEE. Personal use of this material is permitted. However, permission to reprint/republish this material for } \\
\text { advertising or promotional purposes or for creating new collective works for resale or redistribution to servers or lists, } \\
\text { or to reuse any copyrighted component of this work in other works must be obtained from the IEEE. } \\
\text { IEEE, IEEE Transactions on Biomedical Engineering, 35(5), 1988, p296-302 }\end{array}$ \\
\hline Tyре & article \\
\hline File Information & ITBE35_5.pdf \\
\hline
\end{tabular}

Instructions for use 


\section{Visualization of Electric Fields Around a Biological Body}

KOICHI SHIMIZU, MEMBER, IEEE, HIDETO ENDO, AND GORO MATSUMOTO, FELlOW, IEEE

\begin{abstract}
A system was developed which can yisualize the spatial distribution of the ELF (extremely low frequency) electric field around an object with a complex shape such as a biological body. To minimize the field perturbation, an optical field sensor was used. A mechanical $X-Y$ scanner controlled by a microcomputer moves the sensor, automatically scanning the space around the object. The measured data are processed and the field distribution is represented in a color distribution pattern. Using an object with a simple shape such as a cylinder, the accuracy of the measurement was confirmed by comparison to a numerical calculation. The field distributions around experimental animals (a rat and a cat) were measured and it was shown that a conductor model can be used instead of a living body in the ELF range. The field distribution around a human model could be measured in various postures. The results were compared to those of the measurement with a real human body standing under transmission lines, and reasonable agreement was obtained. This technique will provide useful information for the study of the biological effects of the electric field.
\end{abstract}

\section{INTRODUCTION}

A LTHOUGH much effort has been expended on the study of biological effects of microwaves, relatively few studies have been reported in an ELF (extremely low frequency) range until very recently. As the sources of strong electromagnetic energy have increased in our living environment (e.g., high-voltage transmission lines), their biological effects have become a matter of public concern [1]-[4]. When a biological body enters a uniform electric field, the field distribution is perturbed and the field concentrates on some parts of the body in a manner characteristic to the shape of the biological body. In order to analyze the biological effects of electromagnetic energy, the interactions between the electromagnetic field and a biological body have to be well understood. However, there seem to be few techniques available which directly evaluate the electric field exposure on a human body without perturbing the field to be measured. Even with the techniques of numerical analysis, it is not easy to obtain the field distribution around a three-dimensional body with a complex shape such as a human. Some approximated the field distribution by a two-dimensional distribution [5], some assumed a biological body to be of axial symmetry [6], and some simulated a body structure by a collection of small elements such as blocks [7]. They have

Manuscript received December 18, 1985; revised September 25, 1987. This work was supported in part by the Department of Education under Grant-in-Aid for Scientific Research 57850012, 57350020, and 58750018. The authors are with the Research Institute of Applied Electricity, Hokkaido University, Sapporo 060, Japan.

IEEE Log Number 8819586. provided useful information for the understanding of the behavior of the field around a biological body. However, a direct measurement is ultimately required to obtain the field distribution quantitatively around a three-dimensional, nonsymmetrical, smoothly curved biological body. The difficulty of the field measurement is attributed to the lack of a proper instrument. Since most of the field meters currently available are based on the electric principle, they inevitably include metallic parts in the probes and the cables [8], [9]. This causes perturbation of the field around the point to be measured. This restriction makes the field measurement with high spatial resolution difficult. To solve these problems, an optical field sensor was introduced for the measurement with minimum perturbation of the field [10]. Using the optical sensor, a system was developed which measures and visualizes the electric field around an object in a color graphic pattern.

\section{METHOD}

Fig. 1 shows the schematic diagram of the experimental system. The $50 \mathrm{~Hz}$ unperturbed electric field was generated between two plane parallel electrodes. Since in the ELF range, a biological body is considered to be a conductor and the scaling effect can be neglected [11], the object with a conductive surface was used. The object was placed at the center of the lower electrode, and the field distribution around the object was measured.

In the measurement, the equipment and the measurer must be as far away as possible from the electrodes to avoid dangerous high voltage and so as not to distort the field distribution. Furthermore, the position of the field sensor has to be controlled precisely. To satisfy these requirements, a microcomputer-controlled automatic scanning system to carry the sensor was developed. The system first memorizes the contour of the object in the memory of the microcomputer and then scans the space around the object without touching either the object or the electrodes. An optical field sensor which consists of nonmetallic components was connected to the scanner by a dielectric pipe.

Under the command of the microcomputer, the output of the field meter is sampled and recorded after simple data processing, such as time averaging. The measurement is repeated three times with the sensor in three different orientations orthogonal to each other. The magnitude and the orientation of the electric field vector are calculated at each point using these three data. By scan- 
pipes). The probe is moved by a pipe-driving mechanism energized by two pulse motors. The position of the probe is controlled by the number of the pulses sent from a microcomputer with an accuracy of less than $1 \mathrm{~mm}$.

The microcomputer system consists of a microcomputer, a disk drive, a color display, and interfaces. The function of this system is outlined below.

1) The parameters for measurement are entered into the microcomputer via a keyboard. These include the extent of scanning, sampling interval, etc.

2) The outline of the object is obtained by following the outer contour of the object by moving the sensor in a manual control mode performed on the keyboard.

3) A field is generated and an automatic measurement starts. The measurement routine is as follows.

a) The microcomputer waits $1 \mathrm{~s}$ to stop the vibration of the sensor caused by the scanning movement.

b) The output of the field meter is sampled according to predetermined conditions such as sampling frequency.

c) The average and the standard deviation of the sampled data are calculated, and checked to determine if the data are within error limits or not.

d) The probe is moved to the next measurement point.

Steps a)-d) are repeated until the sensor scans all the defined space.

4) After the measurement, all the data are stored on a floppy disk.

5) The data are then processed. The distributions of the field strength and the field orientation are calculated. The results are presented in a color graphic pattern. (In the figures in this paper, they are presented in a monochromatic color.)

Of these steps, 3) and 4) are performed automatically.

\section{Results AND Discussion}

\section{Field Around a Cylinder}

The accuracy of the measurement was checked by comparing the results against theoretical values. Even with a numerical analysis technique, the calculation of the field around a general three-dimensional object is not an easy task. Therefore, for simplicity of calculation, a cylinder was used as a subject. Fig. 3 shows the field distribution around a cylinder obtained by a numerical calculation, i.e., the finite difference method [12]. The field is represented by equipotential lines and electric lines of force. Dense equipotential lines correspond to a strong electric field, and the convergence of electric lines of force indicates the field concentration. As to the vertical component of the electric field, a field concentration on the top of the cylinder, and a weakened field at the side of the cylinder are evident. As to the horizontal component of the field, a concentration occurred around the upper part of the side wall of the cylinder.

Fig. 4(a)-(c) are the results of the measurement. A dielectric cylinder $(20 \mathrm{~cm}$ in diameter and $30 \mathrm{~cm}$ in height) covered with an aluminum foil was placed in a uniform

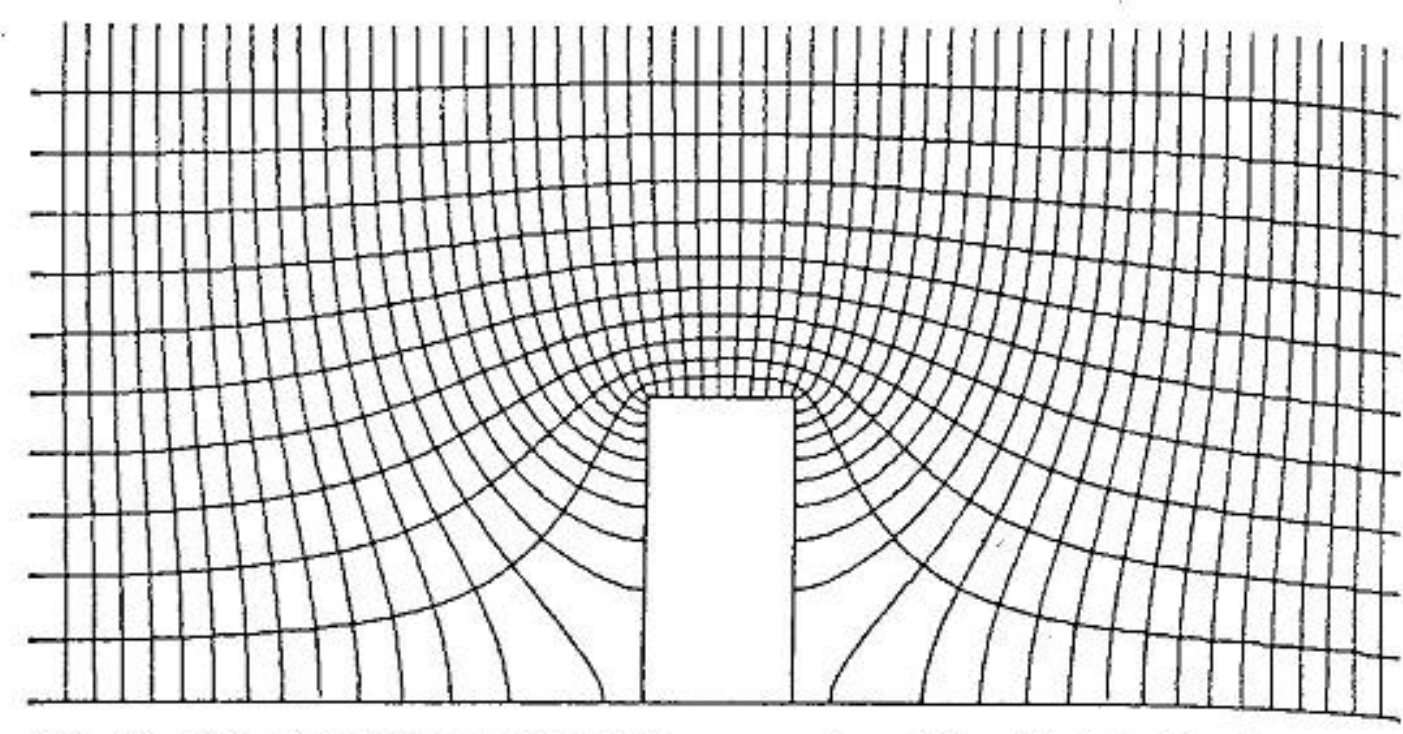

Fig. 3. Calculated field distribution around a solid cylindrical body: equipotential lines (horizontal lines) and lines of electric force (vertical lines).

vertical electric field $(50 \mathrm{~Hz}, 50 \mathrm{kV} / \mathrm{m})$, and the field distributions around it were measured. They are the magnitude distributions of a vertical component [Fig. 4(a)], a horizontal component [Fig. 4(b)], and a depth component [Fig. 4(c)] of the electric field, respectively. The figures are photographs of the images which appeared on a CRT display. The scale of the field strength is presented in the figure in a color code. The maximum value of the scale is adjusted to coincide with the maximum value of the measurement. Using these data, the distribution of the field strength and that of the field orientation were obtained. They are shown in Fig. 4(d)-(e), respectively. The two types of the color code in the figures of the magnitude and the orientation represent the field strength in $\mathrm{kV} / \mathrm{m}$ and the field orientation in degrees, respectively. In the latter case, the horizontal direction is set to be 0 and the vertical direction is 90 . A strong field concentration around the top of the cylinder and a field weakening around the foot of the cylinder are clearly seen. Also, the manner of the field distortion, i.e., the place and the extent of the distortion, are obviously visualized.

Fig. 5 shows the comparison of the measured values to a theoretical calculation. The sensor was scanned along the horizontal line above the top of the cylinder. The agreement between them was excellent (within 10 percent).

\section{Field Around Biological Bodies}

Figs. 6 and 7 show the distributions of the magnitude (a) and the orientation (b) of the electric field around a rat and a cat in the $50 \mathrm{~Hz}$ vertical unperturbed field of 20 $\mathrm{kV} / \mathrm{m}$. In the previous experiments, it was confirmed that the field distribution is independent of the strength of the applied electric field as long as the field is stronger than $S / N$ threshold of the sensor. Thus, in the following experiments, the applied field is set to be $20 \mathrm{kV} / \mathrm{m}$ which is safer than $50 \mathrm{kV} / \mathrm{m}$ used above. In the figures, the part around the object distinguished by hatching is the area where the field sensor cannot reach. The animals were anesthetized by an injection with Nembutal and fixed on the lower electrode. The comparison between Figs. 6 and 7 reveals that the field distribution differs significantly according to the shapes and the postures of the object. That is, the field distortion appears in the vicinity of the body, 


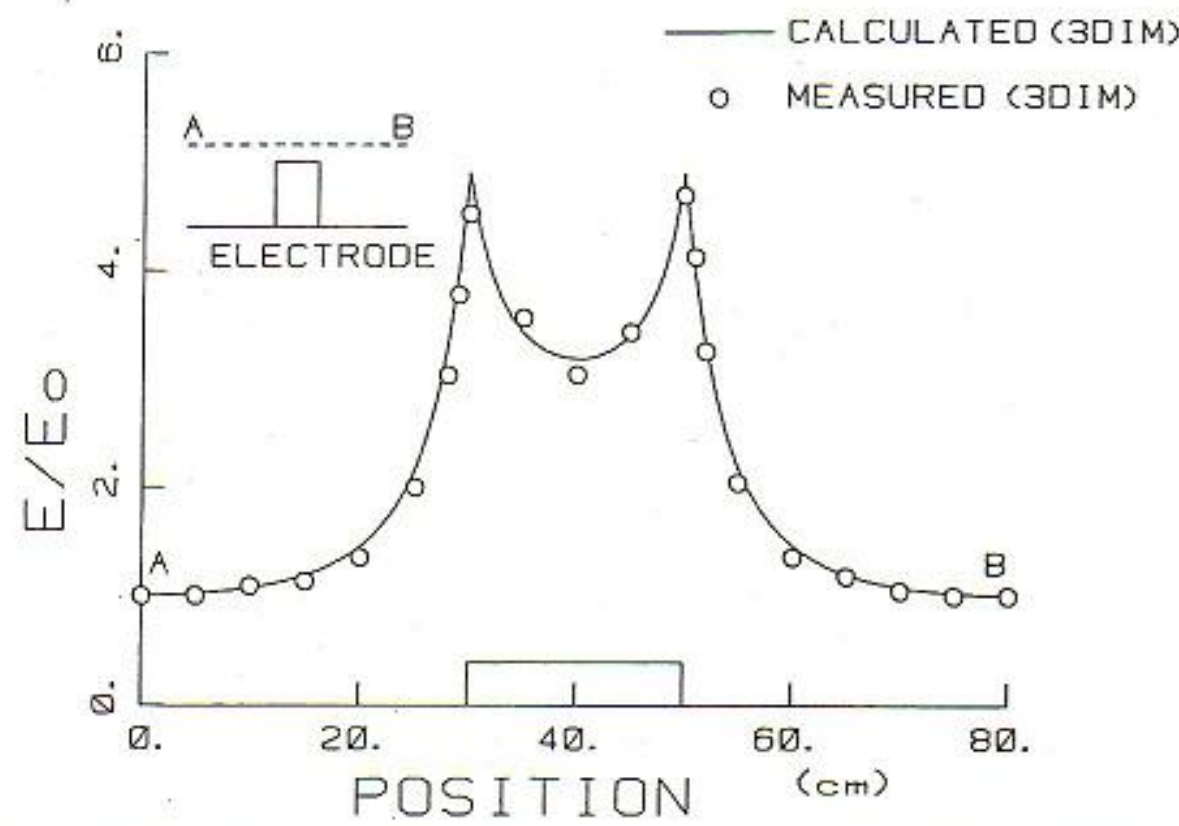

Fig. 5. Comparison between measurement and calculation: horizontal scan.

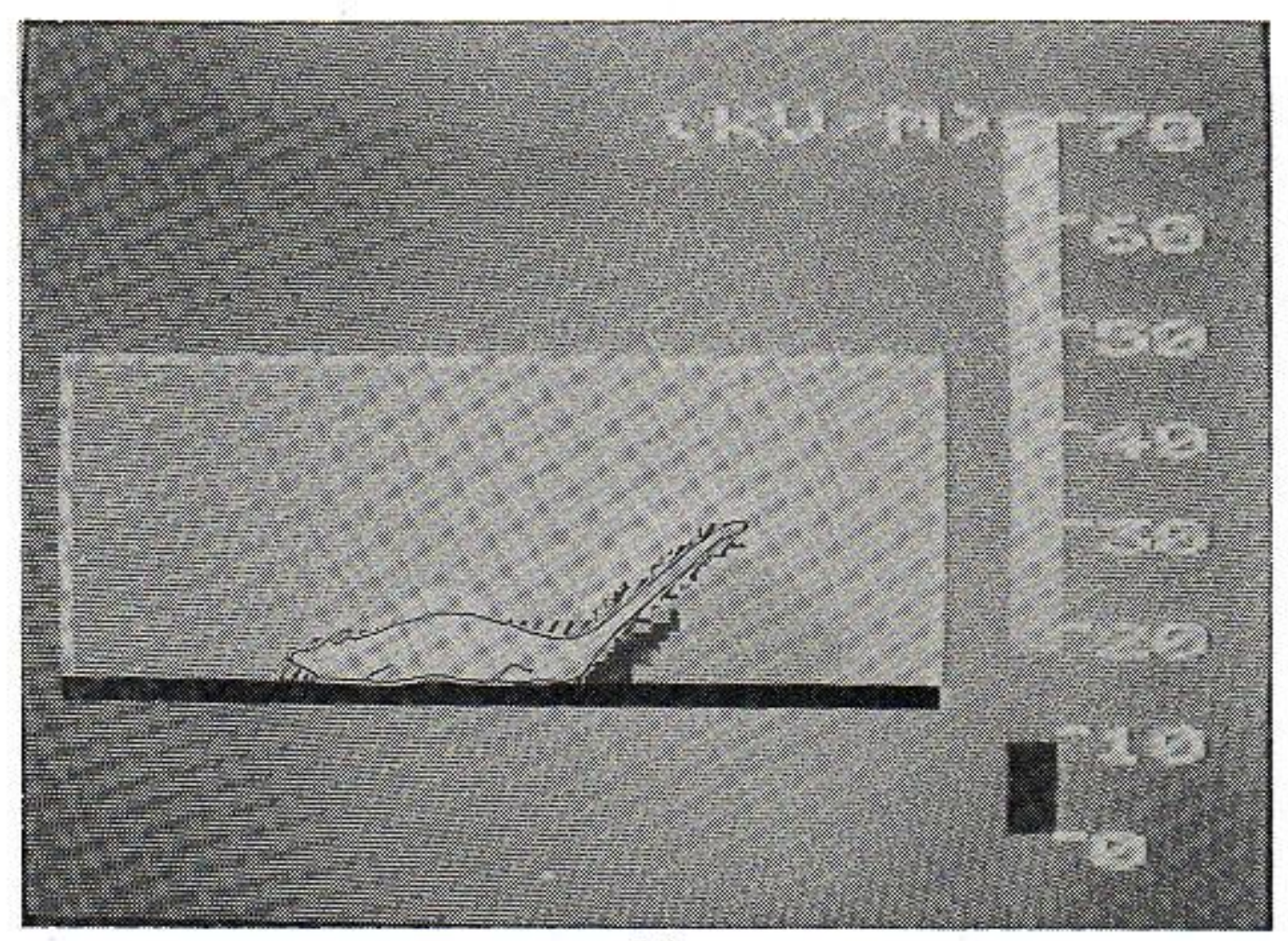

(a)

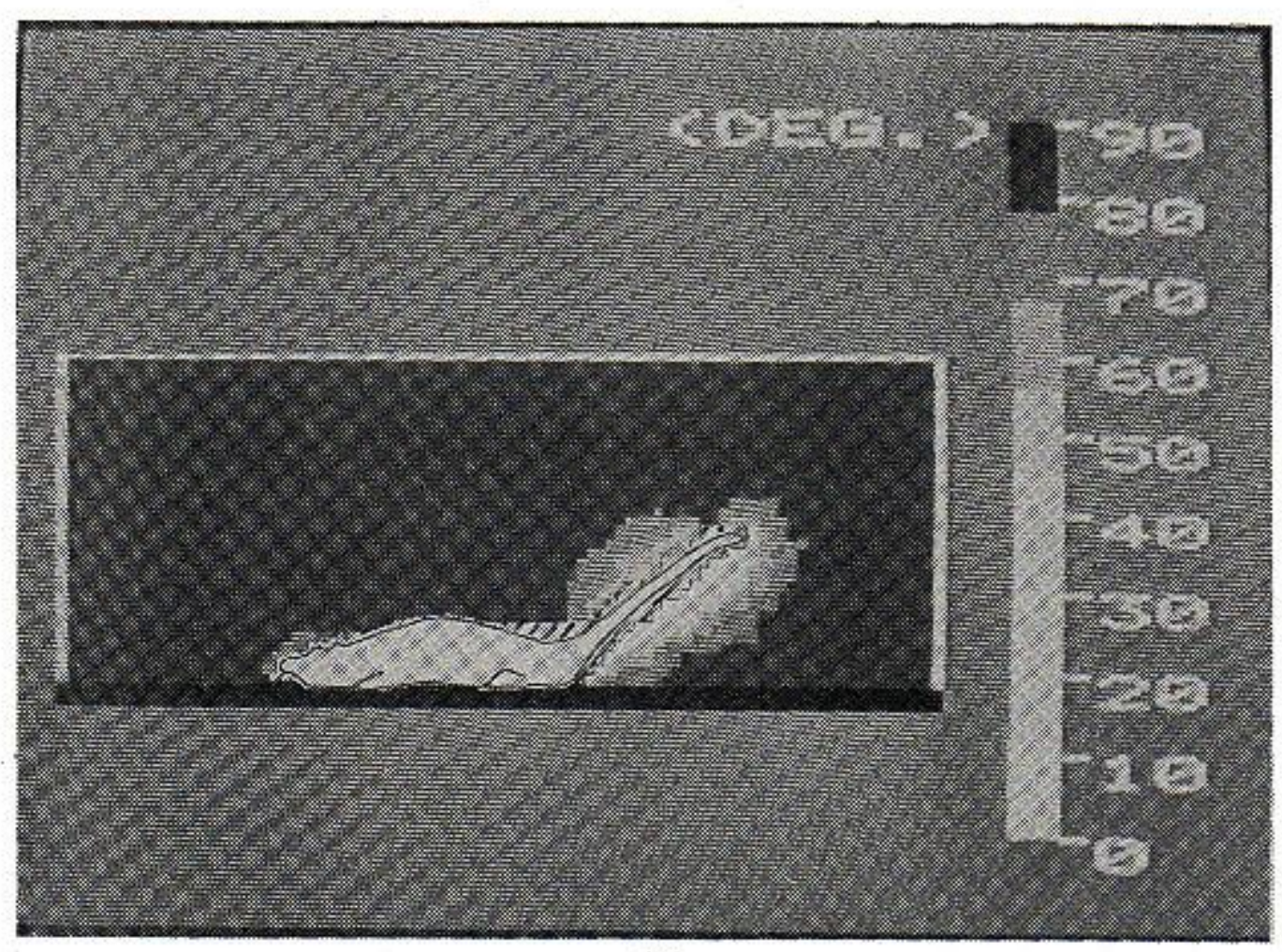

(b)

Fig. 6. Distribution of the magnitude (a) and the orientation (b) of electric field around a rat.

and the characteristic field concentration occurs around the head, back, and tail of the animals.

In order to check the feasibility of using a conductor model instead of a biological body, the field distributions around a rat covered with aluminum foil were compared to those of Fig. 6. There was no difference observed be-

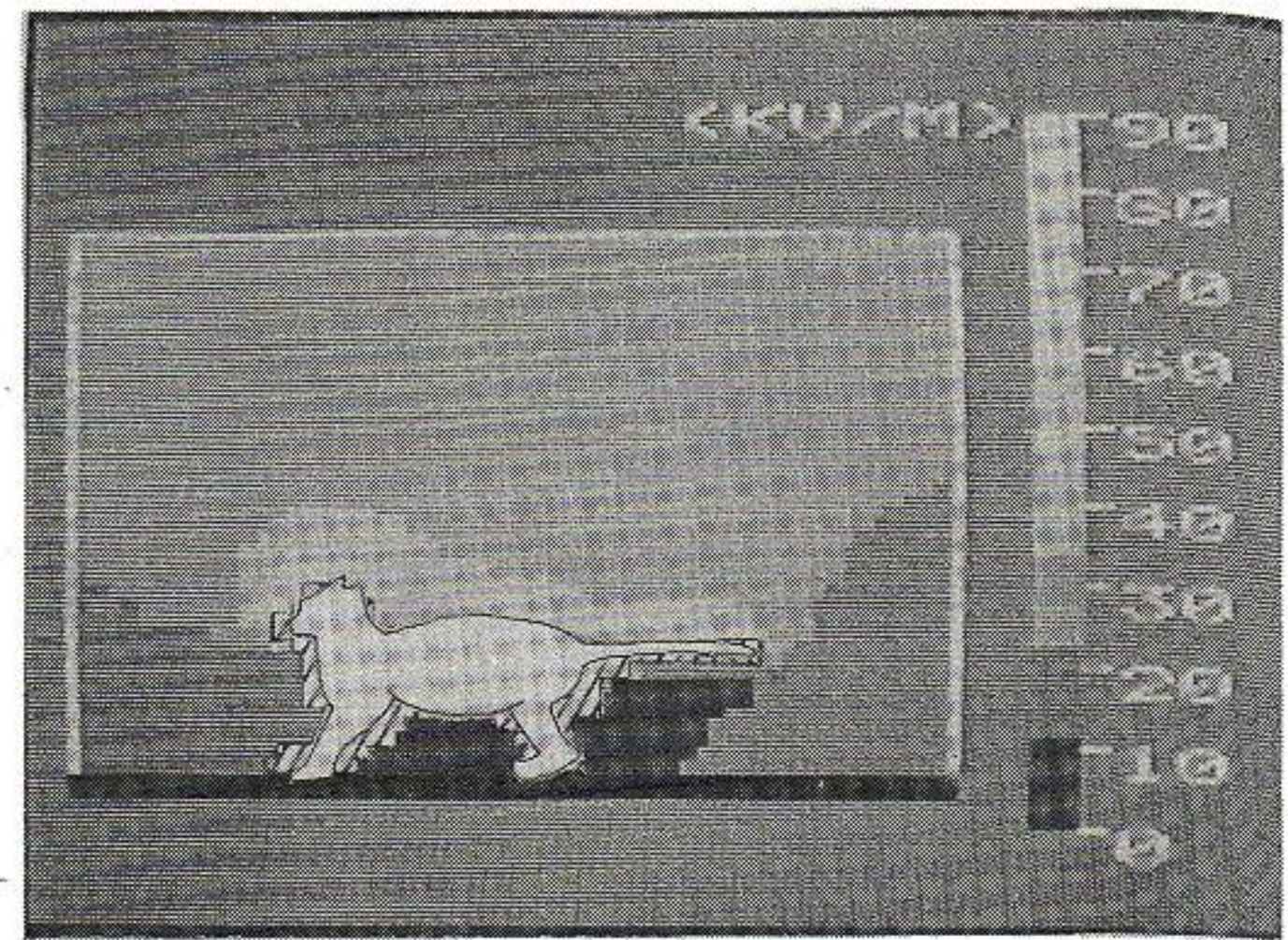

(a)

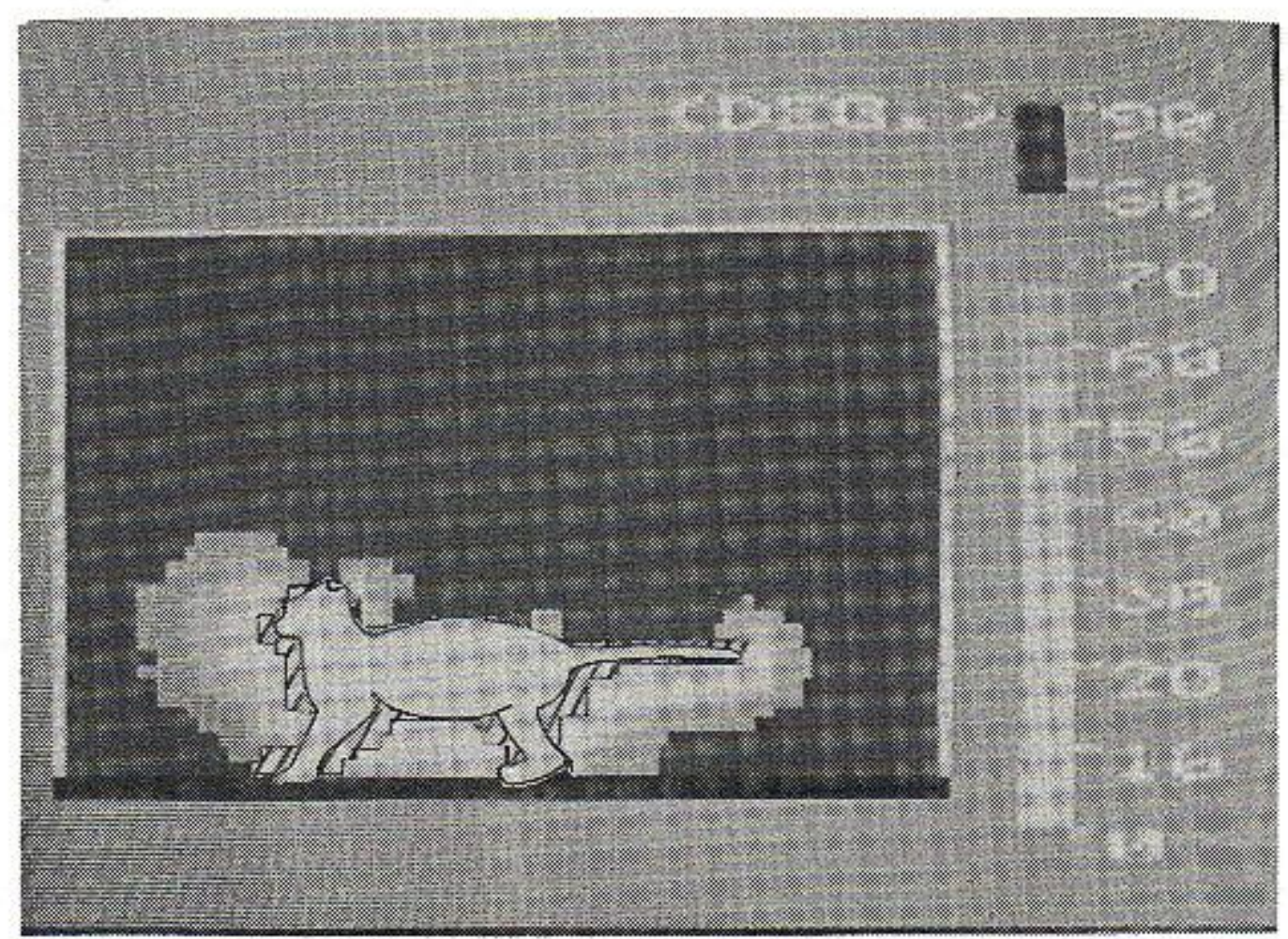

(b)

Fig. 7. Distribution of the magnitude (a) and the orientation (b) of electric field around a cat.

tween the results of a rat with and without a conducting surface. Therefore, it was verified within the accuracy of this measurement that a biological body is considered to be a conductor in low frequency such as 50 or $60 \mathrm{~Hz}$. This result provides a kind of proof for the validity of the usage of a conducting model instead of a biological body.

\section{Field Around a Human Model and a Human}

After checking the validity of this technique, it was applied to the measurement of the electric field around a human model. A dielectric human model ( $40 \mathrm{~cm}$ in height) covered with aluminum foil was used in the measurements.

Figs. 8 and 9 show the distributions of the magnitude of the electric field around a human model placed in the $50 \mathrm{~Hz}$ vertical unperturbed field of $20 \mathrm{kV} / \mathrm{m}$. They are 1) standing posture with the feet grounded (Fig. 8), and 2) standing posture with the feet insulated from the ground (Fig. 9). Comparing Figs. 8 and 9, a significant difference was observed around the side of the body and around the feet. In Fig. 8, the field concentration occurs only at the 
TABLE I

Measured Field Strength at the Points Shown in Fig. 10: $E_{0}=3.5$ $\mathrm{kV} / \mathrm{m}$

\begin{tabular}{|c|l|c|}
\hline \multirow{2}{*}{ point } & \multicolumn{2}{|c|}{ E/Eo } \\
\cline { 2 - 3 } & grounded & insulated \\
\hline A & 6.20 & 4.12 \\
B & 3.60 & 2.17 \\
C & 3.02 & 1.83 \\
D & 0.62 & 0.52 \\
E & 0.50 & 0.60 \\
F & 0.52 & 2.12 \\
G & 0.54 & 1.90 \\
\hline
\end{tabular}

$(E O=3.5 \mathrm{kV} / \mathrm{m})$

logical body using an optical sensor and a microcomputercontrolled automatic scanning system. The accuracy of the measurement using this system was checked by comparing the results to those of numerical calculations. The field distributions around experimental animals were obtained, and it was shown that the distributions differ significantly according to the shapes and the postures of the object. It was also shown that a conductor model can be used instead of a biological body in the ELF range to evaluate the field distribution around the object. The field distributions around a human model were visualized, and the reasonability of the results was confirmed in the measurement with a real human body standing under transmission lines. Through these applications, it was shown that this technique will provide a useful tool for the study on the biological effects of the electromagnetic field. Although the examples presented here are for the evaluation of the field coupling of high-voltage transmission lines, the application of this technique is not necessarily limited to this case. One should be aware that the electrooptic sensor is usable at the frequencies as high as the microwave range. It should be also noted that this technique has enabled us to see a previously invisible quantity, namely, the electric field. The real value of this progress is as yet unknown, but it seems that at least it could promote understanding of the behavior of electric fields, particularly as regards their coupling to other objects.

\section{ACKNOWLEDGMENT}

The authors wish to thank Dr. H. Nakamura of the Central Research Institute of Electric Industry for his help and useful advice, M. Kikuchi and Y. Suzuki of the Hokkaido Electric Industry for providing the field generating system, M. Ihara and K. Tsujii of Sumitomo Electric Industries, Inc. for providing the field sensor, and Dr. T. Kobayashi of Hokkaido Institute of Technology for his help in numerical calculations.

\section{REFERENCES}

[1] A. R. Sheppard and M. Eisenbud, Biological Effects of Electric and Magnetic Field of Extremely Low Frequency. New York: New York Univ. Press, 1977.

[2] R. D. Phillips, M. F. Gillis, W. T. Kaune, and D. D. Mahlum, "Biological effects of extremely low frequency electromagnetic fields," Nat. Tech. Inform. Serv., Springfield, VA,1979.

[3] J. E. Bridges and M. Preache, "Biological influences of power frequency electric fields,"'Proc. IEEE, vol. 69, pp. 1092-1120, 1981.
[4] M. G. Morgan, H. K. Florig, I. Nair, and D. Lincoln, "Powerline fields and human health," IEEE Spectrum, vol. 22, pp. 62-68, 1985.

[5] T. Kobayashi, K. Shimizu, and G. Matsumoto, "Visualization of the electric field around a moving animal by numerical calculation," Trans. IECE Japan, vol. E-65, pp. 565-571, 1982.

[6] Y. Shiau and A. R. Valentino, "ELF electric field coupling to dielectric spheroidal models of biological objects,"' IEEE Trans. Biomed. Eng., vol. BME-28, pp. 429-437, 1981.

[7] R. J. Spiegel, "Numerical determination of induced currents in humans and baboons exposed to $60-\mathrm{Hz}$ electric fields," IEEE Trans. Electromagn. Compat., vol. EMC-23, pp. 382-390, 1981.

[8] C. J. Miller, "The measurement of electric fields in live line working," IEEE Trans. Power App. Syst., vol. PAS-86, pp. 493-498, 1967.

[9] M. Misakian, F. R. Kotter, and R. L. Kahler, "Miniature ELF electric field probe,'" Rev. Sci. Instr., vol. 49, pp. 933-935, 1978.

[10] Y. Hamazaki et al., "OPSEF: An optical measurement of high electric field intensity," Electron. Lett., vol. 16, pp. 406-407, 1980.

[11] W. T. Kaune and M. F. Gillis, "General properties of the interaction between animals and ELF electric fields," Bioelectromagn., vol. 2, pp. $1-11,1981$.

[12] T. Kobayashi et al., " Uniform DC electric field exposure systems for mice and cats," Bioelectromagn., vol. 4, pp. 303-314, 1983.

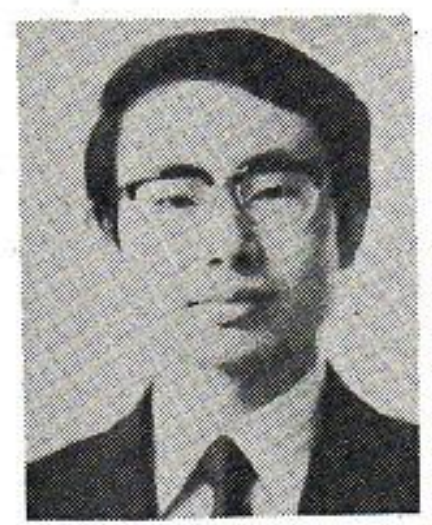

Koichi Shimizu (S'74-M'79) was born in Otaru, Japan, in 1950. He received the B.S. degree from Hokkaido University, Sapporo, Japan. In 1976 and 1979 he received the M.S. and Ph.D. degrees, respectively, from the University of Washington, Seattle.

From 1974 to 1979 he was a Research Associate in the Center for Bioengineering and the Department of Electrical Engineering, University of Washington. He is currently a Research Associate in the Department of Bioengineering, Hokkaido University, Sapporo, Japan. He has been engaged in studies of biomedical engineering including those of wave propagation in biological media, optical biotelemetry, and biological effects of electromagnetic field.

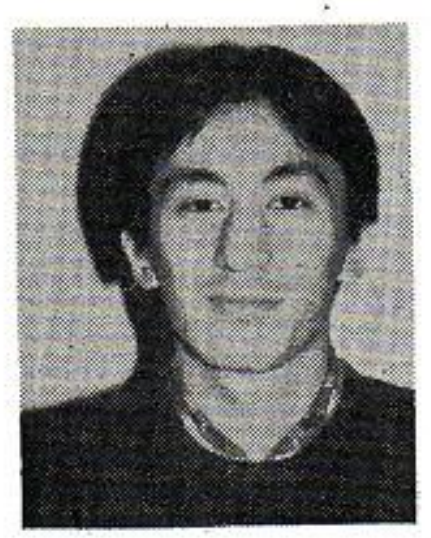

Hideto Endo was born in Obihiro, Japan, in 1959. $\mathrm{He}$ received the B.E. degree in electrical engineering from Towa University, Fukuoka, Japan, in 1982, and the M.E. and Ph.D. degrees in bioengineering from Hokkaido University, Sapporo, Japan, in 1984 and 1987, respectively.

$\mathrm{He}$ is currently with the Suzuye \& Suzuye patent attorney office.

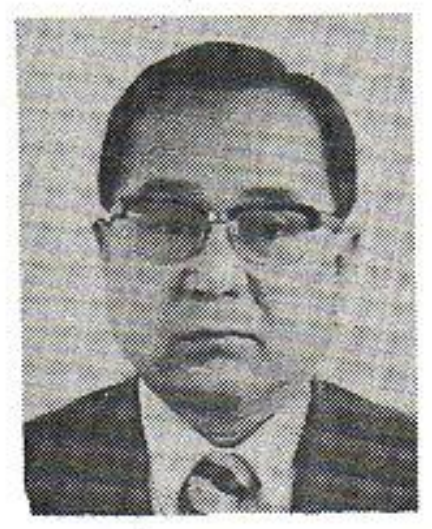

Goro Matsumoto (M'60-SM'83-F'87) received the B.S. and Ph.D. degrees in electrical communication engineering from Tohoku University, Sendai, Japan, in 1949 and 1960, respectively.

From 1949 to 1965 he was a Research Associate and an Associate Professor at Tohoku University. From 1965 to 1987 he was a Professor in the Research Institute of Applied Electricity, Hokkaido University, Sapporo, Japan. In 1987 he was granted the title of a Professor Emeritus in Hokkaido University. He is currently a Professor at the Hokkaido Institute of Technology, Sapporo, Japan. His research interest includes electrical contacts, biomedical instrumentation, biotelemetry, and bioelectromagnetics.

Dr. Matsumoto is a member of the Institute of Electronics and Communication Engineers of Japan, the Japan Society of Applied Physics, the Medical and Biological Engineers of Japan, and the International Society on Biotelemetry. 